East European Journal of Physics

East Eur. J. Phys. 1. 75-85(2019)

DOI: $10.26565 / 2312-4334-2019-1-07$

PACS: $61.72 . \mathrm{Cc}$, 61.72.Mm, 61.72.Qq, 68.35.Dv, 68.35.Fx, 81.10.Jt, 81.30.Mh

\title{
SURFACE-KINETICS-LIMITED OSTWALD RIPENING OF SPHERICAL PRECIPITATES AT GRAIN BOUNDARIES
}

\author{
DOleksandr Koropov*, (D)Roman Skorokhod \\ Institute of Applied Physics of NAS of Ukraine \\ 58, Petropavlivska St., 40000 Sumy, Ukraine \\ *E-mail:ipfmail@ipfcentr.sumy.ua \\ Received 13 November 2018; revised February 28 2019; accepted March 4, 2019
}

Ostwald ripening of sufficiently large (usually macroscopic) precipitates is the late stage of the diffusion decomposition of a supersaturated solid solution, occurring through the formation of fluctuations and subsequent growth of centers (nuclei) of a new phase. The paper describes a theoretical study of the Ostwald ripening of spherical precipitates of a newly formed phase at the grain boundary of finite thickness with the diffusion of impurity atoms from the grain interior to the grain boundary considered. The precipitate growth is assumed to be limited by the kinetics of impurity atom imbedding into the precipitate rather than by the impurity atom diffusion inside the grain boundary. The speed of diffusion growth of spherical precipitate located on the grain boundary is found. A system of equations which describes surface-kinetics-limited growth of Oswald ripening of spherical precipitates on the grain boundary is formulated. This system consists of the equation of growth rate of the precipitate, the kinetic equation for the precipitates size distribution function which is normalized by the precipitates density, and the equation of the balance of matter in the system (the law of conservation of matter). The law of conservation of matter takes into account the atoms of impurities which are in solid solutions of the grain boundary and the body of the grain as well as in the precipitates which is the specifics of our problem. The asymptotic time dependences are found for the average and critical precipitate radius, supersaturation of solid solution of impurity atoms in the grain boundary, precipitate size distribution function, precipitate density, and for the factor of grain boundary filling with precipitates (the area covered by the precipitates per unit area of the grain boundary) and the total number of impurity atoms in precipitates. The factor of grain boundary filling with precipitates is a characteristic of the twodimensional Ostwald ripening problem. A discussion of the limits of validity of obtained results is given.

KEY WORDS: precipitates, Ostwald ripening, grain boundaries, diffusion, surface kinetics.

\section{ВИЗРІВАННЯ ОСТВАЛЬДА СФЕРИЧНИХ ВИДІЛЕНЬ НА МІЖЗЕРЕННІЙ МЕЖІ, ЯКЕ ЛІМІТУСТЬСЯ ПОВЕРХНЕВОЮ КІНЕТИКОЮ \\ О.В. Коропов, Р.В. Скороход \\ Інститут прикладної фізики НАН Украӥни, \\ вул. Петропавлівська, 58, м. Суми 40000, Украӥна}

Визрівання Оствальда достатньо великих (звичайно макроскопічних) виділень - це остання, так звана пізня стадія дифузійного розпаду пересиченого твердого розчину, що відбувається шляхом флуктуаційного утворення і наступного росту центрів (зародків) нової фази. Теоретично розглянуто визрівання Оствальда сферичних виділень нової фази, розташованих на міжзеренній межі скінченної товщини, з урахуванням дифузійних потоків атомів домішки з глибини зерна до міжзеренної межі. Знайдено швидкість дифузійного росту сферичного виділення нової фази, розташованого на міжзеренній межі. Вважається, що ріст виділень лімітується поверхневою кінетикою вбудови атомів домішки в виділення, а не дифузією атомів домішки в міжзеренній межі. Сформульовано систему рівнянь, що описує визрівання Оствальда сферичних виділень, розташованих на міжзеренній межі, ріст яких лімітується поверхневою кінетикою. Ця система складається із рівняння швидкості росту окремого виділення, кінетичного рівняння для функції розподілу виділень нової фази за розмірами, нормованої на густину виділень та рівняння балансу речовини в системі (закону збереження речовини). В законі збереження речовини ураховуються атоми домішки, які знаходяться як в твердих розчинах міжзеренної межі і тіла зерна, так і в виділеннях нової фази, що є специфікою даної задачі. Знайдено асимптотичні часові залежності середнього $\mathrm{i}$ критичного радіусів виділення, пересичення твердого розчину атомів домішки в міжзеренній межі, функції розподілу виділень за розмірами, густини виділень, коефіцієнта заповнення міжзеренної межі виділеннями (площі, що покривається виділеннями на одиниці площі міжзеренної межі) та повного числа атомів домішки у виділеннях. Коефіцієнт заповнення міжзеренної межі виділеннями $\epsilon$ характеристикою, специфічною для двовимірних задач визрівання Оствальда. Обговорюються границі застосування проведеного розгляду.

КЛЮЧОВІ СЛОВА: виділення нової фази, визрівання Оствальда, межі зерен, дифузія, поверхнева кінетика.

\section{СОЗРЕВАНИЕ ОСТВАЛЬДА СФЕРИЧЕСКИХ ВЫДЕЛЕНИЙ НА МЕЖЗЕРЕННОЙ ГРАНИЦЕ, ЛИМИТИРУЕМОЕ ПОВЕРХНОСТНОЙ КИНЕТИКОЙ \\ А.В. Коропов, Р.В. Скороход \\ Институт прикладной физики НАН Украины \\ ул. Петропавловская, 58, г. Сумы 40000, Украина}

Созревание Оствальда достаточно больших (обычно макроскопических) выделений - это последняя, так называемая поздняя стадия диффузионного распада пересыщенного твердого раствора, происходящего путем флуктуационного образования и последующего роста центров (зародышей) новой фазы. Теоретически рассмотрено созревание Оствальда сферических выделений новой фазы, расположенных на межзеренной границе конечной толщины, с учетом диффузионных потоков атомов примеси из глубины зерна к межзеренной границе. Найдена скорость диффузионного роста сферического выделения новой фазы, расположенного на межзеренной границе. Считается, что рост выделений лимитируется поверхностной 
кинетикой встраивания атомов примеси в выделение, а не диффузией атомов примеси в межзеренной границе. Сформулирована система уравнений, описывающая созревание Оствальда сферических выделений, расположенных на межзеренной границе, рост которых лимитируется поверхностной кинетикой. Эта система состоит из уравнения скорости роста отдельного выделения, кинетического уравнения для функции распределения выделений новой фазы по размерам, нормированной на плотность выделений и уравнения баланса вещества в системе (закона сохранения вещества). В законе сохранения вещества учитываются атомы примеси, которые находятся как в твердых растворах межзеренной границы и тела зерна, так и в выделениях новой фазы, что является спецификой данной задачи. Найдены асимптотические временные зависимости среднего и критического радиусов выделения, пересыщения твердого раствора атомов примеси в межзеренной границе, функции распределения выделений по размерам, плотности выделений, коэффициента заполнения межзеренной границы выделениями (площади, покрываемой выделениями на единице площади межзеренной границы) и полного числа атомов примеси в выделениях. Коэффициент заполнения межзеренной границы выделениями является характеристикой, специфической для двумерных задач созревания Оствальда. Обсуждаются пределы применимости проведенного рассмотрения.

КЛЮЧЕВЫЕ СЛОВА: выделения новой фазы, созревание Оствальда, границы зерен, диффузия, поверхностная кинетика.

Дифузійний розпад пересиченого твердого розчину, що відбувається шляхом флуктуаційного утворення і наступного росту центрів нової фази (зародків) включає три основні стадії: 1. інтенсивне утворення зародків 3 розмірами більшими критичного в умовах постійного достатньо великого пересичення, 2. ріст відокремлених (ізольованих) центрів, тобто їх незалежний ріст, 3. визрівання Оствальда (ВО) або коалесценція Оствальда достатньо великих (звичайно макроскопічних) виділень [1-5]. Остання, так звана пізня стадія [2], була відкрита Оствальдом в 1900 р. [6] і полягає в рості більш крупних виділень за рахунок зменшення в розмірі і повному розчиненні дрібних, при цьому середній розмір виділення монотонно зростає як функція часу $t$, а густина виділень монотонно спадає. На стадії ВО сумарна міжфазна поверхнева енергія системи матриця-виділення нової фази на протязі часу зменшується [2, 4-6].

Основи послідовної і внутрішньо несуперечної теорії ВО були закладені в працях Ліфшиця і Сльозова [7] та Вагнера [8]. Ключовим моментом праць $[7,8]$ являється введення до розгляду самопогодженого середнього поля густини атомів домішки, зумовленого всім ансамблем виділень, з якого, власне кажучи, і відбувається ріст виділень нової фази.

В основоположних працях з теорії ВО $[7,8]$ розглядались сферичні виділення нової фази в ідеальній (не містить дефекти) кристалічній матриці, де швидкість масопереносу визначається або дифузією через об'єм матриці-кристала [7], або кінетикою переходу і вбудови атомів на межі матриця-виділення нової фази (поверхневою кінетикою) [8].

В подальшому розглядались багато які питання теорії ВО: ВО двох- і багатокомпонентних виділень нової фази [2, 9], ВО острівцевих структур на поверхні твердого тіла [10-15], ВО кристалів в рідких розчинах [16], ВО центрів кристалізації в кінетиці кристалізації розтопів [17], ВО у бінарній системі з великою об'ємною часткою нової фази при наявності шумів концентрації та фактора Гіббса-Томпсона [18], ВО у бінарній системі при взаємної дифузії з урахуванням нерівноважних вакансій [19], ВО в гетероструктурах з квантовими точками в умовах змішаного типу дифузії $[15,20]$ і ряд інших питань $[2-5,21-32]$.

На відміну від ідеального кристала, в реальному (містить різні дефекти) кристалі кінетика макродефектів на стадії ВО може визначатися дифузією по дислокаційним трубкам [33-36], або дифузією по межам зерен (міжзеренним межам) полікристалів [2, 4, 5, 37-40].

Важливу роль процеси дифузійного розпаду відіграють в матеріалознавстві (включаючи радіаційне матеріалознавство), являючись однією 3 причин старіння металевих стопів [1, 2, 4, 5, 41].

Метою даної роботи $€$ теоретичний аналіз ВО сферичних виділень нової фази, розташованих на міжзеренній межі скінченної товщини $\delta$ [42-44] з урахуванням дифузійних потоків атомів домішки з глибини зерна до міжзеренної межі. Вважається, що ріст виділень лімітується поверхневою кінетикою вбудови атомів домішки в виділення, а не дифузією атомів домішки в міжзеренній межі. Крім того, на відміну від праць [45-47], взагалі кажучи, не передбачається, що радіус виділень $R$ малий в порівнянні з характерним масштабом довжини задачі $L_{B}$.

\section{ШВИДКІСТЬ ДИФУЗІЙНОГО РОСТУ СФЕРИЧНОГО ВИДІЛЕННЯ НОВОЇ ФАЗИ, РОЗТАШОВАНОГО НА МІЖЗЕРЕННІЙ МЕЖІ}

Рівняння дифузії атомів домішки в міжзеренній межі, що розглядається як однорідний ізотропний шар товщиною $\delta$ [42-44], навколо виділення нової фази з урахуванням дифузійних потоків атомів домішки 3 глибини зерна до міжзеренної межі запишемо у вигляді $[48,49]$

$$
L_{B}^{2} \Delta n_{B}-n_{B}+\overline{n_{B}}=0 .
$$

Тут $n_{B}$ - об’ємна густина атомів домішки в міжзеренній межі (двовимірна густина атомів домішки $\left.n_{S}=n_{B} \delta\right), \overline{n_{B}}$ - густина атомів домішки в міжзеренній межі віддалік від виділення нової фази, $L_{B}-$ характерний масштаб довжини задачі, введений у працях [48, 49]. 
Будемо розглядати сферичне виділення нової фази з радіусом $R=R(t) \quad(R>>\delta)$, яке перетинається міжзеренною межею (див. рис. 1) і вважаємо, що розмір зерна $d$ значно перевищує радіус виділення $(d>>R)$.

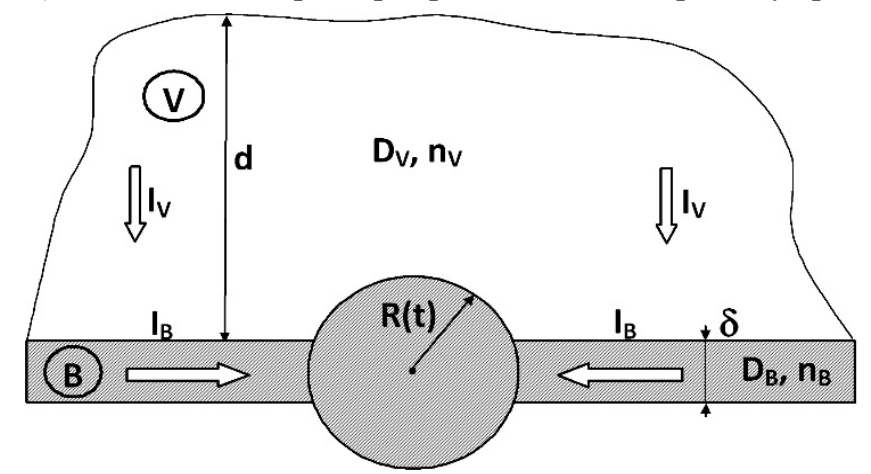

Рис. 1. Схематичне зображення сферичного виділення нової фази, розташованого на міжзеренній межі скінченної товщини $\delta$. $B$ - міжзеренна межа, $V$ - тіло зерна (характерний розмір $d$ ), $D_{V}$ - коефіцієнт дифузії атомів домішки в тілі зерна, $n_{V}$ - густина атомів домішки в тілі зерна, $\mathbf{I}_{V}-$ дифузійний потік атомів домішки в тілі зерна. Зміст останніх позначень пояснюється в тексті.

Граничні умови до рівняння (1) беремо такими [11, 14, 45-49]:

$$
\left.D_{B} \frac{\partial n_{B}}{\partial r}\right|_{r=R}=\beta_{B}\left[n_{B}(R)-n_{B R}\right],\left.\quad n_{B}\right|_{r \rightarrow \infty} \rightarrow \overline{n_{B}} .
$$

Тут $D_{B}$ - коефіцієнт міжзеренної дифузії, тобто об’ємний коефіцієнт дифузії атомів домішки в міжзеренній межі (шарі товщиною $\delta$ ), $\beta_{B}$ - поверхневий кінетичний коефіцієнт, характеризуючий швидкість переходу атомів домішки 3 міжзеренної межі до виділення нової фази, $r$ - відстань від центра виділення в площині міжзеренної межі, $n_{B R}$ - термодинамічно рівноважне значення густини атомів домішки в міжзеренній межі поблизу поверхні міжзеренна межа-виділення $(r=R)$.

Для величини $n_{B R}$ має місце термодинамічна формула

$$
n_{B R}=n_{B \infty} \exp \left(2 \Gamma_{B} / R\right),
$$

де $n_{B \infty}$ - значення $n_{B R}$ поблизу плоскої поверхні міжзеренна межа-виділення $(R \rightarrow \infty), \Gamma_{B} \equiv \sigma_{B} \omega / \kappa T, \sigma_{B}-$ питома міжфазна енергія поверхні міжзеренна межа-виділення, $\omega-$ об’єм, що припадає на один атом домішки у виділенні, $\kappa$ - стала Больцмана, $T$ - температура.

Для подальшого відмітимо, що термодинамічно рівноважний перепад густини атомів домішки в міжзеренній межі поблизу виділення радіуса $R$ такий:

$$
\Delta n_{B R} \equiv \overline{n_{B}}-n_{B R}=n_{B \infty}\left[\exp \left(2 \Gamma_{B} / R^{*}\right)-\exp \left(2 \Gamma_{B} / R\right)\right] .
$$

Тут $R^{*}$ - критичний радіус виділення (виділення з радіусом $R>R^{*}$ зростає, а з радіусом $R<R^{*}$ зменшується в розмірі),

$$
R^{*}=\frac{2 \Gamma_{B}}{\ln \left(\overline{n_{B}} / n_{B \infty}\right)}
$$

Розв'язок рівняння (1), яке задовольняє граничним умовам (2), має вигляд

$$
n_{B}(r)=\overline{n_{B}}-K_{0}\left(r / L_{B}\right)\left[F_{0}\left(R / L_{B}\right)\right]^{-1} \Delta n_{B R},
$$

де введено позначення

$$
F_{0}\left(R / L_{B}\right) \equiv K_{0}\left(R / L_{B}\right)+\left(D_{B} / \beta_{B} L_{B}\right) K_{1}\left(R / L_{B}\right),
$$

$K_{0}\left(K_{1}\right)$ - функція Макдональда нульового (першого) порядку [50].

Швидкість дифузійного росту виділення пропорційна дифузійному потоку атомів домішки в міжзеренній межі $\mathbf{I}_{B}=-D_{B} \nabla n_{B}$ на поверхні міжзеренна межа-виділення $(r=R)$ і знаходиться так:

$$
\frac{d R}{d t}=\left.\frac{D_{B} \omega \delta}{2 R} \frac{d n_{B}(r)}{d r}\right|_{r=R} .
$$


Явний вираз для швидкості росту $d R / d t$ випливає з формул (6), (8) і має вигляд

$$
\frac{d R}{d t}=\frac{D_{B} \omega \delta}{2 R L_{B}} \frac{K_{1}\left(R / L_{B}\right)}{F_{0}\left(R / L_{B}\right)} \Delta n_{B R} .
$$

Якщо ріст виділення лімітується дифузією атомів домішки $\left(\left(D_{B} / \beta_{B} L_{B}\right) K_{1}\left(R / L_{B}\right)<<K_{0}\left(R / L_{B}\right)\right)$, то

$$
\frac{d R}{d t}=\frac{D_{B} \omega \delta}{2 R L_{B}} \frac{K_{1}\left(R / L_{B}\right)}{K_{0}\left(R / L_{B}\right)} \Delta n_{B R} .
$$

Якщо ж ріст виділення лімітується поверхневою кінетикою вбудови атомів домішки у виділення $\left(\left(D_{B} / \beta_{B} L_{B}\right) K_{1}\left(R / L_{B}\right)>>K_{0}\left(R / L_{B}\right)\right)$, то

$$
\frac{d R}{d t}=\frac{\beta_{B} \omega \delta}{2 R} \Delta n_{B R}
$$

Розглянемо тепер інші граничні випадки загального виразу швидкості росту $d R / d t$ (9). Для виділення достатньо великого радіуса $\left(R>>L_{B}\right)$ з формули (9) одержимо

$$
\frac{d R}{d t}=\frac{\overline{D_{B}} \omega \delta}{2 R L_{B}} \Delta n_{B R},
$$

де

$$
\overline{D_{B}} \equiv \frac{D_{B} \beta_{B} L_{B}}{D_{B}+\beta_{B} L_{B}}
$$

ефективний (з урахуванням поверхневої кінетики вбудови) коефіцієнт дифузії атомів домішки в міжзеренній межі. Для виділення малого радіуса $\left(R<L_{B}\right)$ маємо

$$
\frac{d R}{d t}=\frac{D_{B} \omega \delta}{2 R} \frac{\Delta n_{B R}}{R K_{0}\left(R / L_{B}\right)+D_{B} / \beta_{B}} .
$$

Відмітимо, що у випадку $R<<L_{B}$

$$
K_{0}\left(R / L_{B}\right)=-\left[\ln \left(R / 2 L_{B}\right)+\gamma\right]+0\left[\left(R / 2 L_{B}\right)^{2} \ln \left(R / 2 L_{B}\right)\right],
$$

де $\gamma=0.5772 \ldots$ стала Ейлера.

В умовах малих пересичень твердого розчину атомів домішки в міжзеренній межі

$$
\Delta_{B}(t) \equiv \frac{\overline{n_{B}}(t)-n_{B \infty}}{n_{B \infty}}<<1
$$

формула (11) приймає вигляд

$$
\begin{aligned}
\frac{d R}{d t} & =\frac{\beta_{B} \Gamma_{B} n_{B \infty} \omega \delta}{R}\left(\frac{1}{R^{*}}-\frac{1}{R}\right), \\
\frac{d R}{d t} & =\frac{\beta_{B} n_{B \infty} \omega \delta}{2 R}\left(\Delta_{B}-\frac{2 \Gamma_{B}}{R}\right) .
\end{aligned}
$$

Вирази (17), (18) передбачають використання у випадку малих пересичень формул (див. (4), (5))

$$
\begin{gathered}
\Delta n_{B R}=2 \Gamma_{B} n_{B \infty}\left(\frac{1}{R^{*}}-\frac{1}{R}\right), \\
\frac{2 \Gamma_{B}}{R^{*}}=\Delta_{B}<<1 .
\end{gathered}
$$

3 введенням позначення

$$
\mathfrak{B} \equiv \beta_{B} n_{B \infty} \omega \delta,
$$

остаточний вираз (18) для швидкості росту виділення $d R / d t$ запишеться у вигляді

$$
\frac{d R}{d t}=\frac{\mathfrak{B}}{2 R}\left(\Delta_{B}-\frac{2 \Gamma_{B}}{R}\right) .
$$


В цій роботі будемо розглядати ансамбль сферичних виділень, розташованих на міжзеренній межі, ріст яких лімітується поверхневою кінетикою вбудови атомів домішки у виділення за умови малих пересичень твердого розчину атомів домішки в міжзеренній межі $\left(\Delta_{B}<<1\right)$. В цьому випадку швидкість росту виділення $d R / d t$ має вигляд (18), (22).

\section{СИСТЕМА РІВНЯНЬ, ЩО ОПИСУС ВО СФЕРИЧНИХ ВИДІЛЕНЬ, РОЗТАШОВАНИХ НА} МІЖЗЕРЕННІЙ МЕЖІ, РІСТ ЯКИХ ЛІМІТУСТЬСЯ ПОВЕРХНЕВОЮ КІНЕТИКОЮ

В подальшому будемо користуватися безрозмірними величинам: радіусом виділення $\rho \equiv R / R_{0}^{*}=\Delta_{B 0} R / 2 \Gamma_{B} \quad$ і часом $t^{\prime} \equiv t / t_{0}$, де $\left.R_{0}^{*} \equiv R^{*}(t)\right|_{t=0} \quad-$ початковий критичний радіус виділення, $\left.\Delta_{B 0} \equiv \Delta_{B}(t)\right|_{t=0}$ - початкове пересичення атомів домішки в міжзеренній межі, $t_{0} \equiv R_{0}^{* 3} / \mathfrak{B} \Gamma_{B}-$ характерний час задачі. Тоді, опускаючи штрих при $t^{\prime}$, рівняння (22) запишемо у вигляді

$$
\rho^{2} \frac{d \rho}{d t}=\frac{\rho}{x(t)}-1
$$

де

$$
x(t) \equiv R^{*}(t) / R_{0}^{*}=\Delta_{B 0} / \Delta_{B}(t)
$$

безрозмірний критичний радіус виділення, який підлягає визначенню, $x(0)=1$.

Введемо функцію розподілу виділень нової фази за розмірами $f(\rho, t)$, нормовану на густину виділень (число виділень на одиниці площі міжзеренної межі) $\mathcal{N}(t)$, тобто

$$
\mathcal{N}(t)=\int_{0}^{\infty} f(\rho, t) d \rho
$$

$f(\rho, t)$ і $\mathcal{N}(t)$ є шуканими функціями. Функція розподілу $f(\rho, t)$ задовольняє кінетичному рівнянню (рівнянню неперервності в просторі розмірів) $[1,2,7,8]$

$$
\frac{\partial f(\rho, t)}{\partial t}+\frac{\partial}{\partial \rho}\left[f(\rho, t) \frac{d \rho}{d t}\right]=0
$$

з заданою початковою функцією розподілу $\left.f_{0}(\rho) \equiv f(\rho, t)\right|_{t=0}$ і рівнянню балансу речовини в системі (закону збереження речовини)

$$
\overline{n_{B 0}} \delta+\overline{n_{V 0}} d+\frac{4 \pi}{3 \omega} R_{0}^{* 3} \int_{0}^{\infty} \rho^{3} f_{0}(\rho) d \rho=\overline{n_{B}}(t) \delta+\overline{n_{V}}(t) \delta+\frac{4 \pi}{3 \omega} R_{0}^{* 3} \int_{0}^{\infty} \rho^{3} f(\rho, t) d \rho,
$$

де $\left.\overline{n_{B 0}} \equiv \overline{n_{B}}(t)\right|_{t=0}, \overline{n_{V}}(t)$ - густина атомів домішки в глибині зерна, $\left.\overline{n_{V 0}} \equiv \overline{n_{V}}(t)\right|_{t=0}, d-$ характерний поперечний розмір зерна. Відмітимо, що в законі збереження речовини (27) ураховуються атоми домішки, які знаходяться як в твердих розчинах міжзеренної межі і тіла зерна, так і в виділеннях нової фази. Як і раніше [48, 49], будемо вважати, що віддалік від виділення нової фази існує термодинамічна рівновага між атомами домішки, що знаходяться в тілі зерна біля міжзеренної межі і атомами домішки в самій міжзеренній межі. Тоді

$$
\overline{n_{V}}=C \overline{n_{B}}, \quad \overline{n_{V 0}}=C \overline{n_{B 0}},
$$

(формула (10) праці [49]). 3 урахуванням співвідношень (28) закон збереження речовини (27) запишемо у вигляді

$$
Q \equiv \Delta_{B 0}+\lambda^{*} \int_{0}^{\infty} \rho^{3} f_{0}(\rho) d \rho=\Delta_{B}(t)+\lambda^{*} \int_{0}^{\infty} \rho^{3} f(\rho, t) d \rho
$$

де

$$
\lambda^{*} \equiv \frac{4 \pi R_{0}^{* 3}}{3 n_{B \infty} \omega(\delta+C d)}
$$

або ж у вигляді

$$
\frac{\Delta_{B}(t)}{Q}+\lambda \int_{0}^{\infty} \rho^{3} f(\rho, t) d \rho=1 .
$$


Тут

$$
\lambda \equiv \frac{\lambda^{*}}{Q}=\frac{4 \pi R_{0}^{* 3}}{3 n_{B \infty} Q \omega(\delta+C d)}
$$

стала, характеризуюча дану систему. 3 урахуванням формули (24) маємо

$$
\frac{\Delta_{B 0}}{Q x(t)}+\lambda \int_{0}^{\infty} \rho^{3} f(\rho, t) d \rho=1 .
$$

Задача полягає в знаходженні асимптотичного (при $t \rightarrow \infty$ ) розв'язку рівнянь (23), (26) і (33) при заданій початковій функції розподілу $\left.f(\rho, t)\right|_{t=0}=f_{0}(\rho)$. Названі рівняння описують ВО сферичних виділень нової фази, розташованих на міжзеренній межі, ріст яких лімітується поверхневою кінетикою.

\section{АСИМПТОТИКИ ЗМІН КРИТИЧНОГО РАДІУСА ВИДІЛЕННЯ І ПЕРЕСИЧЕННЯ ТВЕРДОГО РОЗЧИНУ АТОМІВ ДОМІШКИ В МІЖЗЕРЕННІЙ МЕЖІ НА СТАДІЇ ВО}

Перейдемо до безрозмірних змінних $u, \tau$ :

$$
\begin{gathered}
u \equiv \frac{R}{R^{*}(t)}=\frac{R}{2 \Gamma_{B}} \Delta_{B}(t)=\frac{\rho}{x(t)}=\frac{\rho}{\Delta_{B 0}} \Delta_{B}(t), \\
\tau \equiv \ln x^{3}=\ln \left(\frac{R^{*}(t)}{R_{0}^{*}}\right)^{3}=\ln \left(\frac{\Delta_{B 0}}{\Delta_{B}(t)}\right)^{3}, \\
x^{3}(\tau)=\exp \tau .
\end{gathered}
$$

В змінних $u, \tau$ рівняння (23) приймає вигляд

$$
\begin{aligned}
& u^{2} \frac{d u}{d \tau}=\frac{1}{3}\left[\mu(u-1)-u^{3}\right], \\
& \mu=\mu(\tau) \equiv \frac{d t}{x^{2} d x}=\frac{3 d t}{d x^{3}}>0 .
\end{aligned}
$$

Якщо через $u(w, \tau)$ позначити розв'язок рівняння (37) при заданій початковій умові $\left.u\right|_{\tau=0}=w$ і урахувати , що $\rho(w, \tau)=x(\tau) u(w, \tau)$ (див. (34)), $\left.\tau\right|_{t=0}=0,\left.x\right|_{\tau=0}=1$ (див. (35), (36)), то повне число атомів домішки в виділеннях всіх розмірів на одиниці площі міжзеренної межі

$$
N_{i}(t)=\frac{4 \pi}{3 \omega} R_{0}^{* 3} \int_{0}^{\infty} \rho^{3} f(\rho, t) d \rho
$$

може бути представлено через початкову функцію розподілу $f_{0}(w)$ у вигляді

$$
N_{i}(\tau)=\frac{4 \pi}{3 \omega} R_{0}^{* 3} x^{3}(\tau) \int_{w_{0}(\tau)}^{\infty} u^{3}(w, \tau) f_{0}(w) d w .
$$

Тут $w_{0}(\tau)$ - розв'язок рівняння $u\left(w_{0}(\tau), \tau\right)=0$, тобто нижня границя первісних радіусів виділень, не розчинених до моменту часу $\tau$. 3 урахуванням формули (39) запишемо закон збереження речовини (33) у вигляді

$$
\frac{\Delta_{B 0}}{Q x(\tau)}+\frac{3 \lambda \omega}{4 \pi R_{0}^{* 3}} N_{i}(\tau)=1
$$

або, остаточно, посилаючись на формули (36) i (40)

$$
1-\frac{\Delta_{B 0}}{Q} \exp (-\tau / 3)=\lambda(\exp \tau) \int_{w_{0}(\tau)}^{\infty} u^{3}(w, \tau) f_{0}(w) d w .
$$

В рівняннях (37) і (42) невідомою функцією являється $\mu(\tau)=d t / x^{2} d x$ і вони повинні бути використані для iii визначення, тобто кінець кінцем для визначення критичного радіуса виділення $x(t)$ і пересичення $\Delta_{B}(t)$.

Аналіз рівнянь (37) i (42), проведений в працях [2, 7], свідчить, що для достатньо великих значень часу $\tau$

$$
\mu(\tau)=\mu_{0}\left(1-3 / 4 \tau^{2}\right), \quad \mu_{0}=27 / 4
$$


і відповідно

$$
x^{3}(t)=\frac{4}{9} t\left[1+\frac{3}{4(\ln t)^{2}}\right] .
$$

Повертаючись до розмірних змінних (критичного радіусу виділення $R^{*}(t)$ і розмірного часу $t$ ), 3 формули (44) одержимо

$$
R^{* 3}=\frac{4}{9} \mathfrak{B} \Gamma_{B} t\left\{1+\frac{3}{4\left[\ln \left(\mathfrak{B} \Gamma_{B} t / R_{0}^{* 3}\right)\right]^{2}}\right\} .
$$

3 формул (43), (45) видно, що основне наближення $\mu(\tau)=\mu_{0}$ і відповідно

$$
R^{* 3}=\frac{4}{9} \mathfrak{B} \Gamma_{B} t=\frac{4}{9} \beta_{B} \Gamma_{B} n_{B \infty} \omega \delta t
$$

справедливе за умови $3 / 4 \tau^{2}<<1$, тобто,

$$
\left[\ln \left(\mathfrak{B} \Gamma_{B} t / R_{0}^{* 3}\right)\right]^{2}>>1
$$

Пересичення твердого розчину атомів домішки в міжзеренній межі асимптотично (при $t \rightarrow \infty$ ) поводить себе наступним чином

$$
\Delta_{B}(t)=\left(\frac{18 \Gamma_{B}^{2}}{\mathfrak{B}}\right)^{1 / 3} t^{-1 / 3}=\left(\frac{18 \Gamma_{B}^{2}}{\beta_{B} n_{B \infty} \omega \delta}\right)^{1 / 3} t^{-1 / 3} .
$$

Зазначимо, що з формул (35) і (46) випливає явна залежність $\tau=\tau(t)$ :

$$
\tau(t)=3 \ln \left(\frac{4 \mathfrak{B} \Gamma_{B}}{9 R_{0}^{* 3}} t\right)=3 \ln \left(\frac{4 \beta_{B} \Gamma_{B} n_{B \infty} \omega \delta}{9 R_{0}^{* 3}} t\right) .
$$

\section{АСИМПТОТИКИ ФУНКЦІї РОЗПОДІЛУ ВИДІЛЕНЬ НОВОЇ ФАЗИ ЗА РОЗМІРАМИ І ГУСТИНИ ВИДІЛЕНЬ НА СТАДІЇ ВО}

Функція розподілу виділень нової фази за розмірами (в змінних $u, \tau) \phi(u, \tau)$ асимптотично (при $\tau \rightarrow \infty$ ) така:

$$
\phi(u, \tau)=A \exp (-\tau) P(u),
$$

де

$$
\begin{gathered}
P(u)=\frac{3^{4} e}{2^{5 / 3}} \frac{u^{2} \exp [-1 /(1-2 u / 3)]}{(u+3)^{7 / 3}(3 / 2-u)^{11 / 3}}, \quad u \in[0 ; 3 / 2), \\
P(u)=0, \quad u \in[3 / 2 ;+\infty) .
\end{gathered}
$$

$P(u) d u \in$ ймовірністю для виділення мати розмір між $u$ та $u+d u$. Відмітимо, що щільність ймовірності $P(u)$ виду (51), (52) була одержана в праці Ліфшиця і Сльозова [7]. Графік функції $P(u)$ представлений рис. 2 (крива 1).

Стала $A$ в формулі (50) визначається законом збереження речовини в системі і дорівнює

$$
A=\left(\lambda \overline{u^{3}}\right)^{-1}
$$

де

$$
\overline{u^{3}}=\int_{0}^{3 / 2} u^{3} P(u) d u \approx 1.1296
$$

Густина виділень нової фази згідно з формулами (25), (50) при $\tau \rightarrow \infty$ наступна

$$
\mathcal{N}=\int_{0}^{\infty} f(\rho, t) d \rho=\int_{0}^{\infty} \phi(u, \tau) d u=A \exp (-\tau) .
$$


3 урахуванням формул (35) і (46) з результату (55) випливає

$$
\mathcal{N}(t)=A\left(\frac{R_{0}^{*}}{R^{*}(t)}\right)^{3}=\frac{9}{4} \frac{A R_{0}^{* 3}}{\beta_{B} \Gamma_{B} n_{B \infty} \omega \delta t} .
$$

В виразі (56), як виходить з формул (32) і (53),

$$
A R_{0}^{* 3}=\frac{R_{0}^{* 3}}{\lambda \overline{u^{3}}}=\frac{3 n_{B \infty} Q \omega(\delta+C d)}{4 \pi \overline{u^{3}}},
$$

тобто асимптотично (при $t \rightarrow \infty) \mathcal{N}(t)$ не залежить від $R_{0}^{*}$.

Оскільки середнє значення $\bar{u}=\bar{R} / R^{*}, \bar{R}(t)=\bar{u} R^{*}(t)$, де $\bar{R}=\bar{R}(t)$ - середній радіус виділення нової фази. Як свідчать праці [2, 7],

$$
\bar{u}=\int_{0}^{3 / 2} u P(u) d u=1
$$

3 щільностю ймовірності $P(u)$ виду (51), (52). Тоді асимптотично (при $t \rightarrow \infty$ )

$$
\bar{R}(t)=R^{*}(t)
$$

тобто,середній радіус виділення збігається з критичним.

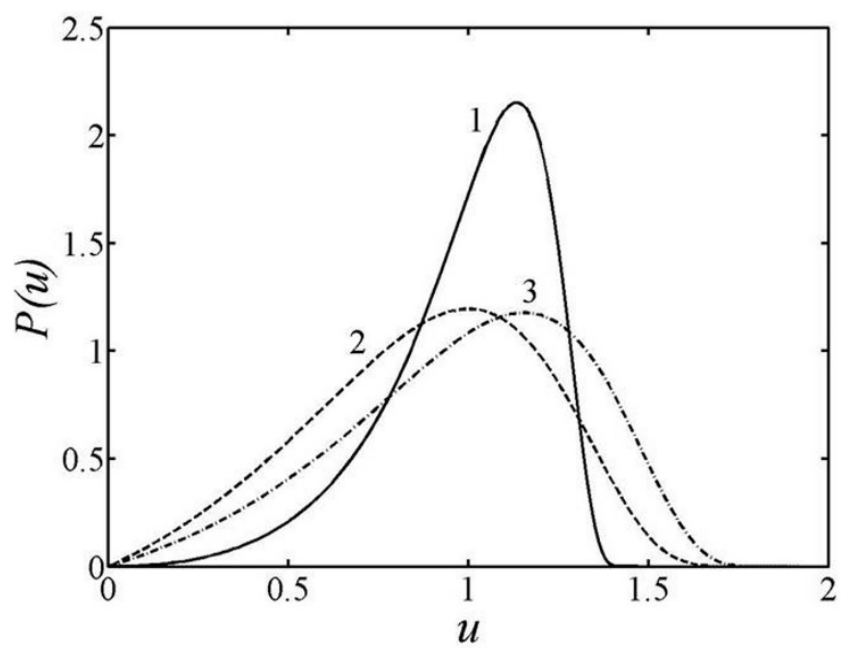

Рис. 2. Деякі функції $P(u)$, одержані в теорії ВО: 1 - праця Ліфшиця і Сльозова [7] та дана робота, 2 - праця Вагнера [8], 3 - праця [39, 40].

Функція розподілу виділень нової фази за розмірами в змінних $R, t$ при $t \rightarrow \infty$ наступна:

$$
\mathcal{F}(R, t)=\frac{\mathcal{N}(t)}{R^{*}(t)} P\left(\frac{R}{R^{*}(t)}\right)=\frac{\mathcal{N}(t)}{\bar{R}(t)} P\left(\frac{R}{\bar{R}(t)}\right) .
$$

Як і в праці [7], асимптотична $(t \rightarrow \infty)$ поведінка функції розподілу виділень за розмірами $\mathcal{F}(R, t)$ не залежить від виду початкової функції розподілу $\mathcal{F}_{0}(R)$. Це зауваження, природно, відноситься і до функції розподілу, записаною в інших змінних, наприклад $\phi(u, \tau)$ (формули (50)-(54)), $f(\rho, t)$.

\section{АСИМПТОТИКИ КОЕФІЩЄНТА ЗАПОВНЕННЯ МІЖЗЕРЕННОЇ МЕЖІ ВИДІЛЕННЯМИ І ПОВНОГО ЧИСЛА АТОМІВ ДОМШКИ У ВИДІЛЕННЯХ НА СТАДІЇ ВО}

Як і в працях $[12-14,39]$, введемо коефіцієнт заповнення міжзеренної межі виділеннями нової фази $\mathcal{Z}(t)$. Саме $\mathcal{Z}(t)$ - це площа, що покривається виділеннями на одиниці площі міжзеренної межі:

$$
\mathcal{Z}(t)=\pi \int_{0}^{\infty} R^{2} \mathcal{F}(R, t) d R=\pi R_{0}^{* 2} \int_{0}^{\infty} \rho^{2} f(\rho, t) d \rho .
$$

3 урахуванням формул (34), (50) i (55) $\mathcal{Z}(t)$ при $t \rightarrow \infty$ запишемо у вигляді 


$$
\mathcal{Z}(t)=\pi\left[R^{*}(t)\right]^{2} \int_{0}^{3 / 2} u^{2} \phi(u, \tau) d u=\pi\left[R^{*}(t)\right]^{2} \mathcal{N}(t) \overline{u^{2}}
$$

де

$$
\overline{u^{2}}=\int_{0}^{3 / 2} u^{2} P(u) d u \approx 1.0463
$$

Оскільки $\mathcal{N}(t)=A\left[R_{0}^{*} / R^{*}(t)\right]^{3}$ (див. (56)), маємо

$$
\mathcal{Z}(t)=\frac{\pi A R_{0}^{* 3} \overline{u^{2}}}{R^{*}(t)} .
$$

Приймаючи до уваги формули (46) i (57), остаточно одержимо

$$
\mathcal{Z}(t)=\left(\frac{3}{2}\right)^{2 / 3} \frac{\pi R_{0}^{* 3} \overline{u^{2}}}{\lambda \overline{u^{3}}}\left(\mathfrak{B} \Gamma_{B} t\right)^{-1 / 3}
$$

Повне число атомів домішки в виділеннях усіх розмірів на одиниці площі міжзеренної межі $N_{i}(t)$ (формула (39)) при $t \rightarrow \infty$ таке:

$$
N_{i}(t)=\frac{4 \pi}{3 \omega}\left[R^{*}(t)\right]^{3 / 2} \int_{0}^{3 / 2} u^{3} \phi(u, \tau) d u=\frac{4 \pi}{3 \omega}\left[R^{*}(t)\right]^{3} \mathcal{N}(t) \overline{u^{3}}
$$

де $\overline{u^{3}}$ надається формулою (54). 3 урахуванням формул (56) i (57)

$$
N_{i}=\frac{4 \pi R_{0}^{* 3}}{3 \lambda \omega}=n_{B \infty} Q(\delta+C d) .
$$

Переконаємося, що саме такий же асимптотичний результат (67) випливає і з закону збереження речовини. Дійсно, оскільки $x(\tau) \rightarrow \infty$ при $\tau \rightarrow \infty, 3$ закону збереження речовини в формі (41) одержимо $N_{i}(\tau) \rightarrow 4 \pi R_{0}^{* 3} / 3 \lambda \omega$ при $\tau \rightarrow \infty .3$ іншого боку, закон збереження речовини у формі (29) з використанням формул (30) і (39) можна представити у вигляді:

$$
Q=\Delta_{B 0}+\frac{N_{i 0}}{n_{B \infty}(\delta+C d)}=\Delta_{B}(t)+\frac{N_{i}(t)}{n_{B \infty}(\delta+C d)},
$$

де $\left.N_{i 0} \equiv N_{i}(t)\right|_{t=0}$. Оскільки $\Delta_{B}(t) \rightarrow 0$ при $t \rightarrow \infty$, із співвідношення (68) маємо $N_{i}(t) \rightarrow n_{B \infty} Q(\delta+C d)$ при $t \rightarrow \infty$, що, природно, збігається з результатом (67).

Таким чином, закон збереження речовини (29), (41) дає такий же саме асимптотичний вираз для повного числа атомів домішки в виділеннях $N_{i}(t)$, як і при безпосередньому обчисленні $N_{i}(t)$ з найденою функцією розподілу $\phi(u, \tau)$ (формула (66)).

Як і в праці [7], представлені вище асимптотичні вирази передбачають виконання нерівності

$$
\tau^{2}=\left(\ln x^{3}\right)^{2}=9\left[\ln \left(\frac{R^{*}(t)}{R_{0}^{*}}\right)\right]^{2}>>1
$$

(див. також нерівність (47), специфічну для даної роботи).

Відзначимо ще, що одержані вище формули 3 деякими змінами можуть бути використаними і для опису процесу ВО сферичних виділень достатньо великого радіуса $\left(R>L_{B}\right)$ для довільного значення $D_{B} / \beta_{B}$. В останньому випадку швидкість росту виділення $d R / d t$ має вигляд (12). Формула (12) формально одержується 3 формули (11) шляхом заміни в (11) $\beta_{B}$ на $\overline{D_{B}} / L_{B}$, а два других рівняння кінетики ВО, саме рівняння (26) i (33) залишаються в силі. Тоді і всі одержані асимптотичні вирази залишаються в силі після заміни $\beta_{B}$ на $\overline{D_{B}} / L_{B}$ в формулах (45) - (49), (56), (65).

\section{ВИСНОВКИ}

Таким чином, теоретично розглянуто процес ВО сферичних виділень нової фази, розташованих на міжзеренній межі скінченної товщини $\delta$ з урахуванням дифузійних потоків атомів домішки з глибини зерна 
характерного розміру $d(d>>>>\delta)$ до міжзеренної межі. Передбачається, що ріст виділень лімітується поверхневою кінетикою вбудови атомів домішки в виділення, що описується феноменологічним поверхневим кінетичним коефіцієнтом $\beta_{B}$ або коефіцієнтом $\mathfrak{B}$, пов'язаним $3 \beta_{B}$ формулою (21). Показано, що такий ріст має місце при виконанні нерівності $\left(D_{B} / \beta_{B} L_{B}\right) K_{1}\left(R / L_{B}\right) \gg>K_{0}\left(R / L_{B}\right)$. У цьому випадку в припущенні малих пересичень твердого розчину атомів домішки в міжзеренній межі $\Delta_{B}(t)<<1$ (нерівність (16)) швидкість росту окремого виділення має вигляд (17), (18), (22). Як і в працях $[2,7,39,40]$, при розгляді ансамблю виділень вважається, що ріст виділень відбувається з самоузгодженого середнього поля атомів домішки, що визначається всім ансамблем виділень.

Знайдені асимптотичні (при $t \rightarrow \infty$ ) часові залежності критичного радіуса виділення $R^{*}(t)$ (формули (44)(47)), пересичення твердого розчину атомів домішки в міжзеренній межі $\Delta_{B}(t)$ (формула (48)), густини виділень $\mathcal{N}(t)$ формула (56)), функції розподілу виділень за розмірами $\phi(u, \tau)$ (формули $(50)-(54)), \mathcal{F}(R, t)$ (формула (60)), коефіцієнта заповнення міжзеренної межі виділеннями $\mathcal{Z}$ (формула (65)), повного числа атомів домішки в виділеннях на одиниці площі міжзеренної межі $N_{i}$ (формула (67)).

Показано, що як і в праці Ліфшиця і Сльозова [7], асимптотично $(t \rightarrow \infty)$ середній радіус виділень дорівнює критичному радіусу $\left(\bar{R}(t)=R^{*}(t)\right)$, а асимптотична поведінка функції розподілу виділень нової фази за розмірами $\mathcal{F}(R, t)$ не залежить від вигляду початкової функції розподілу $\mathcal{F}_{0}(R)$. Стисло обговорені границі застосування проведеного розгляду (див. нерівності (47), (69)).

Показано, що одержані в роботі формули з невеликими змінами придатні для опису ВО сферичних виділень достатньо великого радіуса $\left(R>>L_{B}\right)$ для довільного відношення $D_{B} / \beta_{B}$. Саме всі отримані асимптотичні розв'язки залишаються в силі після заміни $\beta_{B}$ на $\bar{D}_{B} / L_{B}$ в формулах (45)-(49), (56), (65).

\section{ORCID IDs}

Oleksandr Koropov 1 https://orcid.org/0000-0001-9318-1960, Roman Skorokhod 1 https://orcid.org/0000-0002-5951-1070

\section{СПИСОК ЛІТЕРАТУРИ}

[1]. Ya.I. Frenkel', Введение в Теорию Металлов, 4-е изд. [Introduction to the Theory of Metals] (Nauka, Leningrad, 1972), 424 p. (in Russian)

[2]. V.V. Slezov and V.V. Sagalovich, Sov. Phys. Usp. 30(1), 23-45 (1987).

[3]. R.D. Vengrenovitch, Acta Metall. 30(6), 1079-1086 (1982).

[4]. K.V. Chuistov, Упорядочение и Распад в Пересыщенных Твердых Paстворах [Ordering and Decomposition in Supersaturated Solid Solutions] (RIO IMF, Kiev, 1999), 216 p. (in Russian)

[5]. K.V. Chuistov, Старение Металлических Сплавов, 2-е изд. [Aging of Metallic Alloys] (Academperiodika, Kiev, 2003), 568 p. (in Russian)

[6]. W. Ostwald, Zs. Phys. Chem. 34, 495-503 (1900). (in German)

[7]. I.M. Lifshitz and V.V. Slyozov, J. Phys. Chem. Solids. 19(1-2), 35-50 (1961).

[8]. C. Wagner, Zs. Elektrochem. 65(7/8), 581-591 (1961). (in German)

[9]. S.K. Bhattacharyya and K.C. Russell, Metall. Trans. 3(8), 2195-2199 (1972).

[10]. S.A. Kukushkin and A.V. Osipov, Phys. Usp. 41(10), 983-1014 (1998).

[11]. A.V. Koropov and V.V. Sagalovich, Poverkhnost'. Fizika, Khimiya, Mekhanika. No. 6, 50-55 (1987) (in Russian).

[12]. A.V. Koropov and V.V. Sagalovich, Poverkhnost'. Fizika, Khimiya, Mekhanika. No. 5, 55-63 (1989) (in Russian).

[13]. A.V. Koropov and V.V. Sagalovich, Poverkhnost'. Fizika, Khimiya, Mekhanika. No. 2, 17-26 (1990) (in Russian).

[14]. A.V. Koropov, P.N. Ostapchuk and V.V. Slezov, Sov. Phys. Solid State. 33(10), 1602-1607 (1991).

[15]. R.D. Vengrenovich, B.V. Ivanskii and A.V. Moskaliuk, Physics and Chemistry of Solid State. 10(1), 19-30 (2009). (in Ukrainian)

[16]. A.A. Chernov, in: Modern Crystallography III. Crystal Growth, edited by M. Cardona et al. (Springer-Verlag, Heidelberg, 1984), pp. 1-297.

[17]. A.V. Koropov, S.A. Kukushkin and D.A. Grigor'ev, Tech. Phys. 44(7), 786-791 (1999).

[18]. A.M. Gusak and G.V. Lutsenko, Phil. Mag. 85(10), 1323-1331 (2005).

[19]. A.M. Gusak, G.V. Lutsenko and K.N. Tu, Acta Mater. 54(3), 785-791 (2006).

[20]. B.V. Ivanskii, R.D. Vengrenovich, V.I. Kryvetskyi and Yu.M. Kushnir, J. Nano- Electron. Phys. 9(2), 02025 (2017). (in Ukrainian)

[21]. V.V. Slezov, J. Phys. Chem. Solids. 39(4), 367-374 (1978).

[22]. J.A. Marqusee and J. Ross, J. Chem. Phys. 80(1), 536-543 (1984).

[23]. M. Marder, Phys. Rev. A. 36(2), 858-874 (1987).

[24]. P.G. Cheremskoi, V.V. Slezov and V.I. Betekhtin, Поры в Твердом Теле [Pores in Solids] (Energoatomizdat, Moscow, 1990), 376 p. (in Russian)

[25]. B. Giron, B. Meerson and P.V. Sasorov, Phys. Rev. E. 58(4), 4213-4216 (1998).

[26]. V.V. Slezov and J. Schmelzer, Phys. Rev. E. 65, 031506 (2002).

[27]. V.V. Slezov, J. Colloid Interface Sci. 255(2), 274-292 (2002). 
[28]. P. Guyot, L. Lae and C. Sigli, in: Thermodynamics, Microstructures and Plasticity, edited by A. Finel et. al. (Kluwer Academic Publishers, Dordrecht, 2003), pp. 107-121.

[29]. A. Onuki, Phase Transition Dynamics (Cambridge, Cambridge University Press, 2004), 724 p.

[30]. A.S. Shirinian and M.P. Kudyn, Metallofiz. Noveishie Tekhnol. 29(11), 1537-1553 (2007). (in Russian)

[31]. A.S. Shirinian and M.P. Kudyn, Ukr. J. Phys. 53(1), 50-60 (2008). (in Ukrainian)

[32]. R.D. Vengrenovich, B.V. Ivanskii and M.O. Stasyk, Metallofiz. Noveishie Tekhnol. 32(8), 1085-1104 (2010). (in Ukrainian)

[33]. H. Kreye, Zs. Metallkunde. 61(2), 108-112 (1970). (in German)

[34]. A.J. Ardell, Acta Metall. 20(4), 601-609 (1972).

[35]. R.D. Vengrenovich, Yu.V. Gudyma and S.V. Yarema, Phys. Met. Metallogr. 91(3), 228-232 (2001).

[36]. R.D. Vengrenovich, A.V. Moskalyuk and S.V. Yarema, Phys. Solid State. 49(1), 11-17 (2007).

[37]. H.O.K. Kirchner, Metall. Trans. 2(10), 2861-2864 (1971).

[38]. J.W. Martin, R.D. Doherty and B. Cantor, Stability of Microstructure in Metallic Systems, $2^{\text {nd }}$ ed. (Cambridge University Press, Cambridge, 1997), $426 \mathrm{p}$.

[39]. O.V. Koropov, J. Nano- Electron. Phys. 6(1), 01025 (2014). (in Ukrainian)

[40]. O.V. Koropov, in: П'ятнадията Міжнародна Наукова Конференція ім. акад. Михайла Кравчука [Fifteenth International Scientific Mykhailo Kravchuk Conference] (NTUU “KPI”, Kyiv, 2014), 1, pp. 162-167. (in Ukrainian)

[41]. V.N. Voyevodin and I.M. Neklyudov, Эволюиия Структурно-Фазового Состояния и Радиационная Стойкость Конструкиионных Материалов [Evolution of the Structure Phase State and Radiation Resistance of Structural Materials] (Nauk. Dumka, Kiev, 2006), 376 p. (in Russian)

[42]. B.S. Bokstein, Ch.V. Kopezky and L.S. Shvindlerman, Термодинамика и Кинетика Грании Зерен в Металлах [Thermodynamics and Kinetics of Grain Boundaries in Metals] (Metallurgiya, Moscow, 1986), 224 p. (in Russian)

[43]. I. Kaur, Y. Mishin and W. Gust, Fundamentals of Grain and Interphase Boundary Diffusion, $3^{\text {rd }}$ rev. and enl. ed. (John Wiley \& Sons Ltd., New York, 1995), 512 p.

[44]. B.S. Bokstein and A.B. Yaroslavtsev, Диффузия Атомов и Ионов в Твердых Tелах [Diffusion of Atoms and Ions in Solids] (MISIS, Moscow, 2005), 362 p. (in Russian)

[45]. A.V. Koropov, Visnyk of Sumy State University. Seriya: Fizyka, Matematyka, Mekhanika. 9(93), 49-62 (2006). (in Russian)

[46]. A.V. Koropov, Phys. Solid State. 50(11), 2184-2189 (2008).

[47]. A.V. Koropov, J. Surf. Investig. X-Ray, Synchrotron and Neutron Techniques. 5(4), 780-786 (2011).

[48]. A.V. Koropov, J. Nano- Electron. Phys. 2(4), 31-46 (2010).

[49]. A.V. Koropov, Tech. Phys. 56(12), 1781-1786 (2011).

[50]. F. Olver, in: Справочник по специальным функииям с формулами графиками и математическими таблицами [Handbook of Mathematical Functions with Formulas, Graphs and Mathematical Tables] (Transl. from Engl.), edited by M. Abramowitz and I.A. Stegun (Nauka, Moscow, 1979), pp. 177-253. (in Russian) 